\title{
ON TENSOR PRODUCTS OF WEAK MIXING VECTOR SEQUENCES AND THEIR APPLICATIONS TO UNIQUELY $E$-WEAK MIXING $C^{*}$-DYNAMICAL SYSTEMS
}

\author{
FARRUKH MUKHAMEDOV
}

(Received 19 April 2011)

\begin{abstract}
We prove that, under certain conditions, uniform weak mixing (to zero) of the bounded sequences in Banach space implies uniform weak mixing of their tensor product. Moreover, we prove that ergodicity of tensor product of the sequences in Banach space implies their weak mixing. As applications of the results obtained, we prove that the tensor product of uniquely $E$-weak mixing $C^{*}$-dynamical systems is also uniquely $E$-weak mixing.
\end{abstract}

2010 Mathematics subject classification: primary 46L35; secondary 46L55, 46L51, 28D05, 60J99.

Keywords and phrases: uniform weak mixing, weak mixing, ergodicity, uniquely E-weak mixing, $C^{*}$-dynamical system.

\section{Introduction}

Let $X$ be a Banach space with dual space $X^{*}$. In what follows, $B_{X}$ denotes the unit ball in $X$, that is, $B_{X}=\{x \in X:\|x\| \leq 1\}$.

Recall that a sequence $\left\{x_{k}\right\}$ in $X$ is said to be:

(i) weakly mixing to zero if

$$
\lim _{n \rightarrow \infty} \frac{1}{n} \sum_{k=1}^{n}\left|f\left(x_{k}\right)\right|=0 \quad \text { for all } f \in X^{*} ;
$$

(ii) uniformly weakly mixing to zero if

$$
\lim _{n \rightarrow \infty} \sup \left\{\frac{1}{n} \sum_{k=1}^{n}\left|f\left(x_{k}\right)\right|: f \in B_{X^{*}}\right\}=0 ;
$$

(iii) weakly ergodic if

$$
\lim _{n \rightarrow \infty} \frac{1}{n}\left|\sum_{k=0}^{n-1} f\left(x_{k}\right)\right|=0 \quad \text { for all } f \in X^{*}
$$

(C) 2011 Australian Mathematical Publishing Association Inc. 0004-9727/2011 \$16.00 
(iv) ergodic if

$$
\lim _{n \rightarrow \infty} \frac{1}{n}\left\|\sum_{k=0}^{n-1} x_{k}\right\|=0 .
$$

From the definitions one can see that uniform weakly mixing implies weakly mixing, as well as ergodicity implies weak ergodicity. But the converse is not true.

ExAmple 1.1 [20]. Let $X=L^{2}([0,1])$ and $1=n_{1}<n_{2}<\cdots$ be a sequence in $\mathbb{N}$ such that

$$
\frac{n_{j}-1}{n_{j+1}-1} \leq \frac{1}{2}, \quad j \in \mathbb{N}
$$

(for example, $n_{1}=1, n_{2}=2$ and $n_{j+1}=2 n_{j}-1$ for $j \geq 2$ ). Let

$$
1>t_{1}>t_{2}>\cdots>0, \quad t_{j} \rightarrow 0
$$

be real numbers and $g_{j}:[0,1] \rightarrow[0, \infty), j \in \mathbb{N}$, be continuous functions such that

$$
\operatorname{supp}\left(g_{j}\right) \subset\left[t_{j+1}, t_{j}\right] \text { and }\left\|g_{j}\right\|_{2}=1
$$

for all $j \in \mathbb{N}$.

Put

$$
f_{k}=g_{j} \quad \text { for } n_{j} \leq k \leq n_{j+1} .
$$

Then $\left(f_{k}\right)_{k \geq 1}$ is a bounded sequence in $L^{2}([0,1])$, which is weakly convergent to zero, and so is weakly mixing to zero, but which is not uniformly weakly mixing to zero.

Recall [20] that a sequence $\left\{x_{k}\right\}$ in a Banach space $X$ is called convex shift-bounded if there exists a constant $c>0$ such that

$$
\left\|\sum_{j=1}^{p} \lambda_{j} x_{j+k}\right\| \leq c\left\|\sum_{j=1}^{p} \lambda_{j} x_{j}\right\|, \quad k \geq 1,
$$

holds for any $p \in \mathbb{N}$ and $\lambda_{1}, \ldots, \lambda_{p} \geq 0$. One can see that every convex shift-bounded sequence is bounded.

Example 1.2. Let $U: X \rightarrow X$ be a power bounded linear operator (that is, the sequence $\left\{\left\|U^{k}\right\|\right\}$ is bounded). Take $x \in X$; then the sequence $\left\{U^{k}(x)\right\}$ is convex shift-bounded.

The following theorem (see [20]) characterizes weak mixing to zero which is a counterpart of the Blum-Hanson theorem $[6,11]$.

Theorem 1.3. For a convex shift-bounded sequence $\left\{x_{k}\right\}$ in a Banach space $X$ the following conditions are equivalent:

(i) $\left\{x_{k}\right\}$ is weakly mixing to zero;

(ii) $\left\{x_{k}\right\}$ is uniformly weakly mixing to zero.

There is also a characterization of uniformly weak mixing to zero by mean ergodic convergence. 
THEOREM 1.4. For a bounded sequence $\left\{x_{k}\right\}$ in a Banach space $X$ the following conditions are equivalent:

(i) $\quad\left\{x_{k}\right\}$ is uniformly weakly mixing (respectively, weakly mixing) to zero;

(ii) for every sequence $k_{1}<k_{2}<\cdots$ in $\mathbb{N}$ with $\sup _{n \in \mathbb{N}} k_{n} / n<+\infty$, the sequence $\left\{x_{k_{n}}\right\}$ is ergodic (respectively, weakly ergodic).

From this theorem we conclude that weakly ergodicity also does not imply ergodicity.

In the papers mentioned above and others related to them (see $[5,7,11,12]$ ), the tensor product of sequences which obey mixing and ergodicity was not considered. Section 2 of this paper is devoted to the extension of the well-known classical results, stating that a transformation is weakly mixing if and only if its Cartesian square is ergodic [1], for the tensor product of sequences in Banach spaces. In Section 3 we provide some applications of the results obtained to uniquely $E$-ergodic, uniquely $E$-weak mixing $C^{*}$-dynamical systems. Note that such dynamical systems were investigated in $[2,9,10,15,16]$.

\section{Weak mixing vector sequences}

Let $X, Y$ be two Banach spaces with dual spaces $X^{*}$ and $Y^{*}$, respectively. Completion of the algebraic tensor product $X \odot Y$ with respect to a cross-norm $\alpha$ is denoted by $X \otimes_{\alpha} Y$. By $\alpha^{*}$ we denote the conjugate cross-norm to $\alpha$ defined on $X^{*} \odot Y^{*}$.

For the dual Banach spaces $X^{*}$ and $Y^{*}$, denote

$$
B_{X^{*}} \odot B_{Y^{*}}=\left\{\sum_{k=1}^{n} \lambda_{k} x_{k} \otimes y_{k} \mid\left\{x_{k}\right\}_{k=1}^{n} \subset B_{X^{*}},\left\{y_{k}\right\}_{k=1}^{n} \subset B_{Y^{*}}, \lambda_{k} \geq 0, \sum_{k=1}^{n} \lambda_{k} \leq 1, n \in \mathbb{N}\right\} .
$$

Denote by $B_{X^{*}} \otimes_{\alpha^{*}} B_{Y^{*}}$ the closure of $B_{X^{*}} \odot B_{Y^{*}}$ with respect to conjugate crossnorm $\alpha^{*}$. One can see that $B_{X^{*}} \otimes_{\alpha^{*}} B_{Y^{*}} \subset B_{\left(X \otimes_{\alpha} Y\right)^{*}}$. In what follows we consider the following two conditions:

(I) $\quad B_{X^{*}} \otimes_{\alpha^{*}} B_{Y^{*}}=B_{\left(X \otimes_{\alpha} Y\right)^{*}}$;

(II) $X^{*} \otimes_{\alpha^{*}} Y^{*}=\left(X \otimes_{\alpha} Y\right)^{*}$.

One has the following result.

Proposition 2.1. Let $X$ and $Y$ be Banach spaces with a cross-norm $\alpha$ such that property (I) holds. Then (II) is satisfied.

Proof. Assume that (I) is satisfied. Now let us take an arbitrary $f \in\left(X \otimes_{\alpha} Y\right)^{*}$, and show that it can be approximated by elements of $X^{*} \otimes_{\alpha^{*}} Y^{*}$. Indeed, denote $g=f /\|f\|$. Then $g \in B_{\left(X \otimes_{\alpha} Y\right)^{*}}$. Due to (I) we conclude that $g \in X^{*} \otimes_{\alpha^{*}} Y^{*}$. Hence, $f=\|f\| g$ belongs to $X^{*} \otimes_{\alpha^{*}} Y^{*}$.

In what follows, for given $r>0$ and $a \in X$, denote

$$
B_{r, X}(a)=\{x \in X:\|x-a\| \leq r\} .
$$


Proposition 2.2. Let $X$ and $Y$ be Banach spaces with a cross-norm $\alpha$. Then property (I) is satisfied if and only if there exist a number $r, 0<r \leq 1$, and an element $y \in X^{*} \otimes_{\alpha^{*}} Y^{*}$ such that

$$
B_{r,\left(X \otimes_{\alpha} Y\right)^{*}}(y) \subset B_{X^{*}} \otimes_{\alpha^{*}} B_{Y^{*}} .
$$

Proof. It is evident that (I) implies the last property, since it is satisfied with $r=1$ and $y=0$. We now prove the reverse implication. To this end, assume that there exist $r_{0}>0$ and an element $y_{0} \in X^{*} \otimes_{\alpha^{*}} Y^{*}$ such that (2.1) holds. We readily see that $y_{0} \in B_{X^{*}} \otimes_{\alpha^{*}} B_{Y^{*}}$. To prove the statement, it is enough to establish that $B_{\left(X \otimes_{\alpha} Y\right)^{*}} \subset$ $B_{X^{*}} \otimes_{\alpha^{*}} B_{Y^{*}}$. Take any $x \in B_{\left(X \otimes_{\alpha} Y\right)^{*}}$. Consider an element $z=y_{0}+r_{0} x$, which clearly belongs to $B_{r_{0},\left(X \otimes_{\alpha} Y\right)^{*}}$. Due to the assumption, we conclude that $z \in B_{X^{*}} \otimes_{\alpha^{*}} B_{Y^{*}}$. Therefore $x=\left(z-y_{0}\right) / r_{0}$ belongs to $B_{X^{*}} \otimes_{\alpha^{*}} B_{Y^{*}}$.

EXAMPLE 2.3. Let us give some more examples which satisfy conditions (I) and (II).

(i) Let $1<p, q<\infty$, with conjugate indices $p^{\prime}, q^{\prime}$ (that is, $p^{\prime}=p /(p-1)$ ). Consider $\ell_{p}, \ell_{q}$. Then for the projective norm $\pi$ one has $\left(\ell_{p} \otimes_{\pi} \ell_{q}\right)^{*}=\ell_{p^{\prime}} \otimes_{\pi^{*}} \ell_{q^{\prime}}$ if and only if $p>q^{\prime}$ (see [17, Corollary 4.24, Theorem 4.21]).

(ii) We give here a sufficient condition to satisfy (II). The proof can be found in [17, Theorem 5.33]. Let $X$ and $Y$ be Banach spaces such that $X^{*}$ has the RadonNikodym property and either $X^{*}$ or $Y^{*}$ has the approximation property. Then

$$
\left(X \otimes_{\epsilon} Y\right)^{*}=X^{*} \otimes_{\pi} Y^{*}
$$

here $\epsilon$ and $\pi$ are the injective and the projective norms, respectively.

Note that more examples can be found in [17].

Theorem 2.4. Let $X$ and $Y$ be two Banach spaces with a cross-norm $\alpha$ such that property (I) is satisfied. Let $\left\{x_{k}\right\}$ be a bounded sequence in X. Then the following assertions are equivalent:

(i) for any bounded sequence $\left\{y_{k}\right\}$ in $Y$, the sequence $\left\{x_{k} \otimes y_{k}\right\}$ in $X \otimes_{\alpha} Y$ is uniformly weakly mixing to zero;

(ii) $\left\{x_{k}\right\}$ is uniformly weakly mixing to zero.

Proof. (i) $\Rightarrow$ (ii). Let us take any nonzero element $y \in Y$. Define a sequence $\left\{y_{k}\right\}$ by $y_{k}=y$ for all $k \in \mathbb{N}$. For this sequence, due to condition (i), we have

$$
\lim _{n \rightarrow \infty} \sup \left\{\frac{1}{n} \sum_{k=1}^{n}\left|f\left(x_{k} \otimes y\right)\right|: f \in B_{\left(X \otimes_{\alpha} Y\right)^{*}}\right\}=0 .
$$

Now take $f=g \otimes h$ with $g \in B_{X^{*}}$ and $h \in B_{Y^{*}}, h(y) \neq 0$. Then, from (2.2), one gets

$$
\lim _{n \rightarrow \infty}\left(\sup _{g \in B_{X^{*}}}\left\{\frac{1}{n} \sum_{k=1}^{n}\left|g\left(x_{k}\right)\right|\right\}\right)|h(y)|=0,
$$

which implies the assertion. 
(ii) $\Rightarrow$ (i). Let $\left\{y_{k}\right\}$ be an arbitrary bounded sequence in $Y$, and $f \in B_{X^{*}}, g \in B_{Y^{*}}$ be any functionals. Then the Schwarz inequality yields

$$
\begin{aligned}
\frac{1}{n} \sum_{k=1}^{n}\left|f\left(x_{k}\right) g\left(y_{k}\right)\right| & \leq \sqrt{\frac{1}{n} \sum_{k=1}^{n}\left|f\left(x_{k}\right)\right|^{2}} \sqrt{\frac{1}{n} \sum_{k=1}^{n}\left|g\left(y_{k}\right)\right|^{2}} \\
& \leq \max _{k}\left\{\left\|y_{k}\right\|\right\}\|g\| \sqrt{\frac{1}{n} \sum_{k=1}^{n}\left|f\left(x_{k}\right)\right|^{2}}
\end{aligned}
$$

Moreover,

$$
\sup _{f \in B_{X^{*}}}\left\{\frac{1}{n} \sum_{k=1}^{n}\left|f\left(x_{k}\right)\right|^{2}\right\} \leq \max \left\{\left\|x_{k}\right\|\right\} \sup _{f \in B_{X^{*}}}\left\{\frac{1}{n} \sum_{k=1}^{n}\left|f\left(x_{k}\right)\right|\right\} \rightarrow 0
$$

as $n \rightarrow \infty$. Therefore, (2.3) implies that

$$
\lim _{n \rightarrow \infty} \sup _{\substack{f \in B_{X^{*}} \\ g \in B_{Y^{*}}}}\left\{\frac{1}{n} \sum_{k=1}^{n}\left|f \otimes g\left(x_{k} \otimes y_{k}\right)\right|\right\}=0 .
$$

Hence, using the norm-denseness of the elements $\sum_{k=1}^{m} \lambda_{k} f_{k} \otimes g_{k},\left\{f_{k}\right\} \subset B_{X^{*}},\left\{g_{k}\right\} \subset$ $B_{Y^{*}}$ (where $\lambda_{k} \geq 0, \sum_{k=1}^{n} \lambda_{k} \leq 1$ ) in $B_{X^{*}} \otimes_{\alpha^{*}} B_{Y^{*}}$, from (2.4) one gets

$$
\lim _{n \rightarrow \infty} \sup _{\varphi \in B_{X^{*}} \otimes_{\alpha^{*}} B_{Y^{*}}}\left\{\frac{1}{n} \sum_{k=1}^{n}\left|\varphi\left(x_{k} \otimes y_{k}\right)\right|\right\}=0 .
$$

Thanks to property (I) one has

$$
\sup _{f \in B_{\left(X \otimes_{\alpha} Y\right)^{*}}}\left\{\frac{1}{n} \sum_{k=0}^{n-1}\left|f\left(x_{k} \otimes y_{k}\right)\right|\right\}=\sup _{w \in B_{X^{*}} \otimes_{\alpha^{*}} B_{Y^{*}}}\left\{\frac{1}{n} \sum_{k=0}^{n-1}\left|w\left(x_{k} \otimes y_{k}\right)\right|\right\} .
$$

Consequently, (2.5) yields the required statement.

REMARK 2.5. From the proof of Theorem 2.4 one can see that the implication (i) $\Rightarrow$ (ii) is still valid without property (I).

Using the same argument as in the proof above, we get the following theorem.

Theorem 2.6. Let $X$ and $Y$ be two Banach spaces with a cross-norm $\alpha$ such that property (II) is satisfied. Let $\left\{x_{k}\right\}$ be a bounded sequence in $X$. Then the following assertions are equivalent:

(i) for any bounded sequence $\left\{y_{k}\right\}$ in $Y$, the sequence $\left\{x_{k} \otimes y_{k}\right\}$ in $X \otimes_{\alpha} Y$ is weakly mixing to zero;

(ii) $\left\{x_{k}\right\}$ is weakly mixing to zero. 
Proposition 2.7. Let $X$ be a Banach space and $\left\{x_{k}\right\}$ be a bounded sequence in $X$ such that the sequence $\left\{x_{k} \otimes x_{k}\right\}$ is ergodic in $X \otimes_{\alpha} X$. Then $\left\{x_{k}\right\}$ is uniformly weakly mixing to zero.

Proof. Ergodicity of the the sequence $\left\{x_{k} \otimes x_{k}\right\}$ means that

$$
\lim _{n \rightarrow \infty} \frac{1}{n}\left\|\sum_{k=1}^{n} x_{k} \otimes x_{k}\right\|=0 .
$$

Due to the equality

$$
\sup _{\mathbf{f} \in B_{\left(X \otimes_{\alpha} Y\right)^{*}}}\left|\mathbf{f}\left(\frac{1}{n} \sum_{k=0}^{n-1} x_{k} \otimes x_{k}\right)\right|=\frac{1}{n}\left\|\sum_{k=1}^{n} x_{k} \otimes x_{k}\right\|,
$$

one finds that

$$
\sup _{f \in B_{X^{*}}}\left\{\frac{1}{n}\left|f \otimes f\left(\sum_{k=0}^{n-1} x_{k} \otimes x_{k}\right)\right|\right\} \leq \frac{1}{n}\left\|\sum_{k=1}^{n} x_{k} \otimes x_{k}\right\| .
$$

On the other hand,

$$
\begin{aligned}
\sup _{f \in B_{X^{*}}}\left\{\frac{1}{n}\left|f \otimes f\left(\sum_{k=0}^{n-1} x_{k} \otimes x_{k}\right)\right|\right\} & =\sup _{f \in B_{X^{*}}}\left\{\frac{1}{n}\left|\sum_{k=0}^{n-1} f \otimes f\left(x_{k} \otimes x_{k}\right)\right|\right\} \\
& =\sup _{f \in B_{X^{*}}}\left\{\frac{1}{n} \sum_{k=0}^{n-1}\left|f\left(x_{k}\right)\right|^{2}\right\},
\end{aligned}
$$

which with (2.6), (2.7) yields

$$
\lim _{n \rightarrow \infty} \sup _{f \in B_{X^{*}}}\left\{\frac{1}{n} \sum_{k=0}^{n-1}\left|f\left(x_{k}\right)\right|^{2}\right\}=0 .
$$

Hence, the Schwarz inequality implies that

$$
\sup _{f \in B_{X^{*}}}\left\{\frac{1}{n} \sum_{k=0}^{n-1}\left|f\left(x_{k}\right)\right|\right\} \leq \sqrt{\sup _{f \in B_{X^{*}}}\left\{\frac{1}{n} \sum_{k=0}^{n-1}\left|f\left(x_{k}\right)\right|^{2}\right\} .}
$$

Therefore, we find that $\left\{x_{k}\right\}$ is uniformly weakly mixing to zero.

Similarly, one can prove the following proposition.

Proposition 2.8. Let $X$ be a Banach space and $\left\{x_{k}\right\}$ be a bounded sequence in $X$ such that the sequence $\left\{x_{k} \otimes x_{k}\right\}$ is weakly ergodic in $X \otimes_{\alpha} X$. Then $\left\{x_{k}\right\}$ is weakly mixing to zero.

Theorem 2.9. Let $X$ be a Banach space with a cross-norm $\alpha$ on $X \odot X$ such that condition (I) is satisfied with $Y=X$. Let $\left\{x_{k}\right\}$ be a be bounded sequence in $X$. Then the following assertions are equivalent: 
(i) the sequence $\left\{x_{k} \otimes x_{k}\right\}$ is ergodic in $X \otimes_{\alpha} X$;

(ii) the sequence $\left\{x_{k} \otimes x_{k}\right\}$ is uniformly weakly mixing to zero in $X \otimes_{\alpha} X$;

(iii) $\left\{x_{k}\right\}$ is uniformly weakly mixing to zero.

Proof. The implication (i) $\Rightarrow$ (iii) immediately follows from Proposition 2.7. The implication (iii) $\Rightarrow$ (ii) follows from Theorem 2.4. The implication (ii) $\Rightarrow$ (i) is evident.

Using the same argument as above in the proof of Theorem 2.6, one gets the following theorem.

Theorem 2.10. Let $X$ be a Banach space with a cross-norm $\alpha$ on $X \odot X$ such that condition (II) is satisfied with $Y=X$. Let $\left\{x_{k}\right\}$ be a bounded sequence in $X$. Then the following assertions are equivalent:

(i) the sequence $\left\{x_{k} \otimes x_{k}\right\}$ is weakly ergodic in $X \otimes_{\alpha} X$;

(ii) the sequence $\left\{x_{k} \otimes x_{k}\right\}$ is weakly mixing to zero in $X \otimes_{\alpha} X$;

(iii) $\left\{x_{k}\right\}$ is weakly mixing to zero.

Theorem 2.11. Let $X$ and $Y$ be two Banach spaces with a cross-norm $\alpha$ on $X \odot Y$ such that condition (I) (respectively, (II)) is satisfied. Let $\left\{x_{k}\right\}$ be a bounded sequence in $X$. The following assertions are equivalent:

(i) for any bounded sequence $\left\{y_{k}\right\}$ in $Y$, the sequence $\left\{x_{k} \otimes y_{k}\right\}$ in $X \otimes_{\alpha} Y$ is ergodic (respectively, weakly ergodic);

(ii) $\left\{x_{k}\right\}$ is uniformly weakly mixing (respectively, weakly mixing) to zero.

Proof. (i) $\Rightarrow$ (ii). Let us take any nonzero element $y \in Y$. Define a sequence $\left\{y_{k}\right\}$ by $y_{k}=y$ for all $k \in \mathbb{N}$. For this sequence, due to condition (i), we have

$$
\lim _{n \rightarrow \infty}\left\|\frac{1}{n} \sum_{k=1}^{n} x_{k} \otimes y\right\|=\lim _{n \rightarrow \infty}\left\|\frac{1}{n} \sum_{k=1}^{n} x_{k}\right\|\|y\|=0,
$$

which means that $\left\{x_{k}\right\}$ is ergodic. The condition yields that $\left\{x_{k} \otimes x_{k}\right\}$ is ergodic, hence Theorem 2.9 implies that $\left\{x_{k}\right\}$ is uniformly weakly mixing to zero.

(ii) $\Rightarrow$ (i). Using Theorem 2.4 , we find that $\left\{x_{k} \otimes y_{k}\right\}$ is uniformly weakly mixing to zero, for every bounded sequence $\left\{y_{k}\right\}$ in $Y$. Hence, it is ergodic.

\section{Applications to $C^{*}$-dynamical systems}

In this section $\mathfrak{A}$ will be a $C^{*}$-algebra with the unity $\mathbb{1}$. Recall that a linear functional $\varphi \in \mathfrak{A}^{*}$ is called positive if $\varphi\left(x^{*} x\right) \geq 0$ for every $x \in \mathfrak{A}$. A positive functional $\varphi$ is said to be a state if $\varphi(\mathbb{1})=1$. We denote by $\mathcal{S}(\mathfrak{A})$ the set of all states in $\mathfrak{A}$. A linear operator $T: \mathfrak{A} \rightarrow \mathfrak{A}$ is called positive if $T x \geq 0$ whenever $x \geq 0$. We denote by $M_{n}(\mathfrak{A})$ the set of all $n \times n$ matrices $a=\left(a_{i j}\right)$ with entries $a_{i j}$ in $\mathfrak{A}$. A linear mapping $T: \mathfrak{A} \rightarrow \mathfrak{A}$ is called completely positive if the linear operator $T_{n}: M_{n}(\mathfrak{A}) \rightarrow M_{n}(\mathfrak{H})$ given by $T_{n}\left(a_{i j}\right)=$ $\left(T\left(a_{i j}\right)\right)$ is positive for all $n \in \mathbb{N}$. A completely positive map $T: \mathfrak{A} \rightarrow \mathfrak{A}$ with $T \mathbb{1}=\mathbb{1}$ is 
called a unital completely positive map. A pair ( $\mathfrak{A}, T)$ consisting of a $C^{*}$-algebra $\mathfrak{A}$ and a unital completely positive map $T: \mathfrak{A} \rightarrow \mathfrak{A}$ is called $a C^{*}$-dynamical system (see [18]). Let $\mathfrak{B}$ be another $C^{*}$-algebra with unit. A completion of the algebraic tensor product $\mathfrak{A} \odot \mathfrak{B}$ with respect to the minimal $C^{*}$-tensor norm on $\mathfrak{A} \odot \mathfrak{B}$ is denoted by $\mathfrak{A} \otimes \mathfrak{B}$, and it would also be a $C^{*}$-algebra with a unit (see [18]). It is known [18] that if (ㄱ, $T$ ) and $(\mathfrak{B}, H)$ are two $C^{*}$-dynamical systems, then $(\mathfrak{H} \otimes \mathfrak{B}, T \otimes H)$ is also a $C^{*}$-dynamical system, since a mapping $T \otimes H: \mathfrak{A} \otimes \mathfrak{B} \rightarrow \mathfrak{A} \otimes \mathfrak{B}$ given by $(T \otimes H)(x \otimes y)=T x \otimes H y$ is a unital completely positive map.

Let $(\mathfrak{A}, T)$ be a $C^{*}$-dynamical system, and $\mathfrak{B}$ be a subspace of $\mathfrak{A}$. Let $E: \mathfrak{A} \rightarrow \mathfrak{B}$ be a norm-one projection, that is $E^{2}=E$. In [8] (see also $[3,9,16]$ ) the following notation is introduced.

Definition 3.1. A $C^{*}$ dynamical system $(\mathfrak{A}, T)$ is said to be:

(i) unique $E$-ergodic if

$$
\lim _{n \rightarrow \infty} \frac{1}{n} \sum_{k=1}^{n} \varphi\left(T^{k}(x)\right)=\varphi(E(x)), \quad x \in \mathfrak{A}, \varphi \in \mathcal{S}(\mathfrak{H}) ;
$$

(ii) unique $E$-weakly mixing if

$$
\lim _{n \rightarrow \infty} \frac{1}{n} \sum_{k=0}^{n-1}\left|\varphi\left(T^{k}(x)\right)-\varphi(E(x))\right|=0, \quad x \in \mathfrak{A}, \varphi \in \mathcal{S}(\mathfrak{U}) .
$$

It can readily be seen (in $[3,9])$ that the map $E$ is a norm-one projection onto the fixed point subspace $\mathfrak{A}^{T}=\{x \in \mathfrak{A}: T x=x\}$. Therefore, in what follows we denote it by $E_{T}$. In [2] (see also [3]), (i) is called unique ergodicity with respect to the fixed point subalgebra, whereas, in [9], (ii) is called E-strictly weak mixing. In addition, when $E=\omega(\cdot) \mathbb{1}$ (that is, when there is a unique invariant state for $T$ ), (i) is the well-known unique ergodicity, and (ii) is called strict (unique) weak mixing [16]. Note that in [4] relations between unique ergodicity, minimality and weak mixing were studied.

By using the Jordan decomposition of bounded linear functionals (see [18]), one can replace $\mathcal{S}(\mathfrak{H})$ with $\mathfrak{A}^{*}$ in Definition 3.1.

Note that in $[9,15]$ it has been shown that the free shift on the reduced amalgamated free product $C^{*}$-algebra, and length-preserving automorphisms of the reduced $C^{*}$ algebra of the $R D$-group for the length function, including the free shift on the free group on infinitely many generators, enjoy a unique $E$-mixing property. Such a class of dynamical systems was defined and studied for the first time in [2]. Note that in [10] other more complicated unique $E$-ergodic and unique mixing $C^{*}$-dynamical systems arising from free probability are studied. Note that in [7] sufficient and necessary conditions for ergodicity in terms of joinings are studied.

In this section we apply the results of the previous section to these concepts.

Theorem 3.2. Let ( $\mathfrak{A}, T),(\mathfrak{B}, H)$ be two $C^{*}$-dynamical systems, and assume that $(\mathfrak{A} \otimes \mathfrak{B})^{*}=\mathfrak{A}^{*} \otimes \mathfrak{B}^{*}$ is satisfied. Then the following assertions are equivalent: 
(i) the $C^{*}$-dynamical system $(\mathfrak{A} \otimes \mathfrak{B}, T \otimes H)$ is unique $E_{T \otimes H}$-weak mixing;

(ii) ( $(\mathfrak{A}, T)$ and $(\mathfrak{B}, H)$ are unique $E_{T}$-weak mixing and $E_{H}$-weak mixing, respectively.

Proof. (i) $\Rightarrow$ (ii). According to the condition for all arbitrary functionals $\psi \in \mathfrak{A}^{*}$ and $\phi \in \mathcal{S}(\mathfrak{B})$,

$$
\begin{aligned}
0 & =\lim _{n \rightarrow \infty} \frac{1}{n} \sum_{k=0}^{n-1}\left|\psi \otimes \phi\left(T^{k} \otimes H^{k}(x \otimes \mathbb{1})\right)-\psi \otimes \phi\left(E_{T \otimes H}(x \otimes \mathbb{1})\right)\right| \\
& =\lim _{n \rightarrow \infty} \frac{1}{n} \sum_{k=0}^{n-1}\left|\psi\left(T^{k}(x)\right)-\psi \otimes \phi\left(E_{T \otimes H}(x \otimes \mathbb{1})\right)\right| .
\end{aligned}
$$

Hence

$$
\lim _{n \rightarrow \infty} \frac{1}{n} \sum_{k=0}^{n-1} T^{k}(x)
$$

converges weakly, and we denote its limit by $E_{T}$. Consequently, from (3.1) one finds that $E_{T \otimes H}(\cdot \otimes \mathbb{1})=E_{T}(\cdot)$. Moreover, $(\mathfrak{A}, T)$ is unique $E_{T}$-weak mixing. Similarly, we get unique $E_{H}$-weak mixing of $(\mathfrak{B}, H)$.

Let us consider the implication (ii) $\Rightarrow(\mathrm{i})$. Let $x \in \mathfrak{A}$ and $y \in \mathfrak{B}$. Define two sequences

$$
x_{k}=T^{k}(x)-E_{T}(x), \quad y_{k}=H^{k}(y)-E_{H}(y), \quad k \in \mathbb{N} .
$$

Then one can see that the sequences are weakly mixing. Hence, Theorem 2.6 implies that the sequence $\left\{x_{k} \otimes y_{k}\right\}$ is weakly mixing as well. This means that for every $\omega \in(\mathfrak{H} \otimes \mathfrak{B})^{*}$,

$$
\begin{aligned}
& \lim _{n \rightarrow \infty} \frac{1}{n} \sum_{k=1}^{n} \mid \omega\left(T^{k}(x) \otimes H^{k}(y)\right)-\omega\left(T^{k}(x) \otimes E_{H}(y)\right) \\
&-\omega\left(E_{T}(x) \otimes H^{k}(y)\right)+\omega\left(E_{T}(x) \otimes E_{H}(y)\right) \mid=0 .
\end{aligned}
$$

Now define two functionals $\omega_{1}$ and $\omega_{2}$ on $\mathfrak{A}$ and $\mathfrak{B}$, respectively, as follows:

$$
\omega_{1}(\cdot)=\omega\left(\cdot \otimes E_{H}(y)\right), \quad \omega_{2}(\cdot)=\omega\left(E_{T}(x) \otimes \cdot\right)
$$

here $E_{T}(x)$ and $E_{H}(y)$ are fixed. Then, according to the weak mixing condition (see (ii)),

$$
\begin{aligned}
& \lim _{n \rightarrow \infty} \frac{1}{n} \sum_{k=1}^{n}\left|\omega_{1}\left(T^{k}(x)\right)-\omega_{1}\left(E_{T}(x)\right)\right|=0, \\
& \lim _{n \rightarrow \infty} \frac{1}{n} \sum_{k=1}^{n}\left|\omega_{2}\left(H^{k}(y)\right)-\omega_{2}\left(E_{H}(y)\right)\right|=0 .
\end{aligned}
$$


Relations (3.5) and (3.6), together with (3.4), mean that

$$
\begin{aligned}
& \lim _{n \rightarrow \infty} \frac{1}{n} \sum_{k=1}^{n}\left|\omega\left(T^{k}(x) \otimes E_{H}(y)\right)-\omega\left(E_{T}(x) \otimes E_{H}(y)\right)\right|=0, \\
& \lim _{n \rightarrow \infty} \frac{1}{n} \sum_{k=1}^{n}\left|\omega\left(E_{T}(x) \otimes H^{k}(y)\right)-\omega\left(E_{T}(x) \otimes E_{H}(y)\right)\right|=0 .
\end{aligned}
$$

The inequality

$$
\begin{aligned}
\mid \omega\left(T^{k} \otimes\right. & \left.H^{k}(x \otimes y)\right)-\omega\left(E_{T}(x) \otimes E_{H}(y)\right) \mid \\
\leq & \mid \omega\left(T^{k}(x) \otimes H^{k}(y)\right)-\omega\left(T^{k}(x) \otimes E_{H}(y)\right) \\
& -\omega\left(E_{T}(x) \otimes H^{k}(y)\right)+\omega\left(E_{T}(x) \otimes E_{H}(y)\right) \mid \\
& +\left|\omega\left(T^{k}(x) \otimes E_{H}(y)\right)-\omega\left(E_{T}(x) \otimes E_{H}(y)\right)\right| \\
& +\left|\omega\left(E_{T}(x) \otimes H^{k}(y)\right)-\omega\left(E_{T}(x) \otimes E_{H}(y)\right)\right|,
\end{aligned}
$$

together with (3.3), (3.7) and (3.8), implies that

$$
\lim _{n \rightarrow \infty} \frac{1}{n} \sum_{k=1}^{n}\left|\omega\left(T^{k} \otimes H^{k}(x \otimes y)\right)-\omega\left(E_{T} \otimes E_{H}(x \otimes y)\right)\right|=0 .
$$

The norm-denseness of the elements $\sum_{i=1}^{m} x_{i} \otimes y_{i}$ in $\mathfrak{A} \otimes \mathfrak{B}$ with (3.9) yields

$$
\lim _{n \rightarrow \infty} \frac{1}{n} \sum_{k=1}^{n}\left|\omega\left(T^{k} \otimes H^{k}(\mathbf{z})\right)-\omega\left(E_{T} \otimes E_{H}(\mathbf{z})\right)\right|=0,
$$

for arbitrary $\mathbf{z} \in \mathfrak{A} \otimes \mathfrak{B}$. So, $(\mathfrak{A} \otimes \mathfrak{B}, T \otimes H)$ is unique $E_{T} \otimes E_{H}$-weak mixing.

Corollary 3.3. Let $(\mathfrak{H}, T)$ and $(\mathfrak{B}, H)$ be unique $E_{T}$-weak mixing and $E_{H}$-weak mixing, respectively. Then one has $E_{T \otimes H}=E_{T} \otimes E_{H}$.

REMARK 3.4. Note that in $[13,19]$ certain spectral conditions of tensor products of dynamical systems defined on von Neumann algebras were studied. We have to stress that in those papers, dynamical systems have faithful normal invariant states. For such weak mixing dynamical systems the condition $E_{T \otimes H}=E_{T} \otimes E_{H}$ is proved as well.

Example 3.5. Now let us provide an example of a $C^{*}$-dynamical system which does not have any invariant faithful state, but where $E_{T \otimes H}=E_{T} \otimes E_{H}$.

Let $\mathfrak{A}=\mathbb{C}^{2}$ and $\mathfrak{B}=\mathbb{C}^{3}$ and

$$
T=\left(\begin{array}{cc}
\frac{1}{2} & \frac{1}{2} \\
0 & 1
\end{array}\right), \quad H=\left(\begin{array}{ccc}
1 & 0 & 0 \\
0 & 1 & 0 \\
0 & \frac{1}{2} & \frac{1}{2}
\end{array}\right)
$$


It is clear that

$$
\begin{gathered}
\mathfrak{A}^{T}=\{(x, x): x \in \mathbb{C}\}, \\
\mathfrak{B}^{H}=\{(x, y, y): x, y \in \mathbb{C}\} .
\end{gathered}
$$

One can check that all invariant states for $H$ have the form

$$
(p, q, 0), \quad p, q \geq 0, p+q=1,
$$

which is not faithful.

Direct calculations show that

$$
\lim _{n \rightarrow \infty} T^{n}(x, y)=E_{T}(x, y), \quad \lim _{n \rightarrow \infty} H^{n}(x, y, z)=E_{H}(x, y, z),
$$

which mean that $T$ and $H$ are unique $E_{T}$-weak mixing and $E_{H}$-weak mixing, respectively. Here

$$
E_{T}(x, y)=(y, y), \quad E_{H}(x, y, z)=(x, y, y) .
$$

Now let us calculate $(\mathfrak{A} \otimes \mathfrak{B})^{T \otimes H}$. To do so, one can see that

$$
T \otimes H=\frac{1}{2}\left(\begin{array}{ll}
H & H \\
0 & 2 H
\end{array}\right) .
$$

Denote $\mathbf{x}=\left(x_{1}, x_{2}, x_{3}\right), \mathbf{y}=\left(y_{1}, y_{2}, y_{3}\right)$. Then from $T \otimes H(\mathbf{x}, \mathbf{y})=(\mathbf{x}, \mathbf{y})$ we find that

$$
\frac{1}{2} H(\mathbf{x}+\mathbf{y})=\mathbf{x}, \quad H \mathbf{y}=\mathbf{y} .
$$

Simple algebra shows that $\mathbf{x}=\mathbf{y}$. Consequently,

$$
(\mathfrak{A} \otimes \mathfrak{B})^{T \otimes H}=\left\{\left(x_{1}, x_{2}, x_{2}, x_{1}, x_{2}, x_{2}\right): x_{1}, x_{2} \in \mathbb{C}\right\},
$$

which yields that $(\mathfrak{A} \otimes \mathfrak{B})^{T \otimes H}=\mathfrak{A}^{T} \otimes \mathfrak{B}^{H}$. This implies that $E_{T \otimes H}=E_{T} \otimes E_{H}$.

Moreover, by the same argument we may show that the equality $E_{H \otimes H}=E_{H} \otimes E_{H}$ holds as well.

REMARK 3.6. The theorem proved above extends some results of $[14,15]$. We note that in $[4,13,19]$ similar results were proved for weak mixing dynamical systems defined over von Neumann algebras.

Note that some examples of $C^{*}$-algebras which satisfy the condition $(\mathfrak{A} \otimes \mathfrak{B})^{*}=$ $\mathfrak{A}^{*} \otimes \mathfrak{B}^{*}$ can be found in [15] (see also [17]).

Theorem 3.7. Let ( $\mathfrak{A}, T)$ be a $C^{*}$-dynamical system. Then for the following assertions:

(i) ( $\mathfrak{A}, T)$ is unique $E_{T}$-weak mixing;

(ii) for every $(\mathfrak{B}, H)$-unique $E_{H}$-ergodic $C^{*}$-dynamical system with $E_{T \otimes H}=E_{T} \otimes E_{H}$ and $\mathfrak{H}^{*} \otimes \mathfrak{B}^{*}=(\mathfrak{H} \otimes \mathfrak{B})^{*}$, the $C^{*}$-dynamical system $(\mathfrak{H} \otimes \mathfrak{B}, T \otimes H)$ is unique $E_{T} \otimes E_{H^{-} \text {ergodic; }}$

the implication $(i) \Rightarrow$ (ii) holds true. 
Proof. Let $(\mathfrak{B}, H)$ be a $C^{*}$-dynamical system as in (ii). Now take arbitrary elements $x \in \mathfrak{A}$ and $y \in \mathfrak{B}$, and consider the corresponding sequences $\left\{x_{k}\right\}$ and $\left\{y_{k}\right\}$ given by (3.2). Then, due to the condition, $\left\{x_{k}\right\}$ is weak mixing and $\left\{y_{k}\right\}$ is weak ergodic. Hence, Theorem 2.11 yields that $\left\{x_{k} \otimes y_{k}\right\}$ is weak ergodic, which means that, for every $\omega \in(\mathfrak{A} \otimes \mathfrak{B})^{*}$,

$$
\begin{gathered}
\lim _{n \rightarrow \infty} \frac{1}{n} \sum_{k=1}^{n}\left(\omega\left(T^{k}(x) \otimes H^{k}(y)\right)-\omega\left(T^{k}(x) \otimes E_{H}(y)\right)\right. \\
\left.-\omega\left(E_{T}(x) \otimes H^{k}(y)\right)+\omega\left(E_{T}(x) \otimes E_{H}(y)\right)\right)=0 .
\end{gathered}
$$

Using similar arguments as in the proof of Theorem 3.2, we find that

$$
\begin{aligned}
& \lim _{n \rightarrow \infty} \frac{1}{n} \sum_{k=1}^{n}\left|\omega\left(T^{k}(x) \otimes E_{H}(y)\right)-\omega\left(E_{T}(x) \otimes E_{H}(y)\right)\right|=0, \\
& \lim _{n \rightarrow \infty} \frac{1}{n} \sum_{k=1}^{n}\left(\omega\left(E_{T}(x) \otimes H^{k}(y)\right)-\omega\left(E_{T}(x) \otimes E_{H}(y)\right)\right)=0 .
\end{aligned}
$$

From

$$
\begin{aligned}
\mid \frac{1}{n} \sum_{k=1}^{n}( & \left.\left(T^{k} \otimes H^{k}(x \otimes y)\right)-\omega\left(E_{T}(x) \otimes E_{H}(y)\right)\right) \mid \\
\leq & \mid \frac{1}{n} \sum_{k=1}^{n}\left(\omega\left(T^{k}(x) \otimes H^{k}(y)\right)-\omega\left(T^{k}(x) \otimes E_{H}(y)\right)\right. \\
& \left.-\omega\left(E_{T}(x) \otimes H^{k}(y)\right)+\omega\left(E_{T}(x) \otimes E_{H}(y)\right)\right) \mid \\
& +\frac{1}{n} \sum_{k=1}^{n}\left|\omega\left(T^{k}(x) \otimes E_{H}(y)\right)-\omega\left(E_{T}(x) \otimes E_{H}(y)\right)\right| \\
& +\left|\frac{1}{n} \sum_{k=1}^{n}\left(\omega\left(E_{T}(x) \otimes H^{k}(y)\right)-\omega\left(E_{T}(x) \otimes E_{H}(y)\right)\right)\right|
\end{aligned}
$$

and using (3.10)-(3.12), we obtain

$$
\lim _{n \rightarrow \infty} \frac{1}{n} \sum_{k=1}^{n}\left(\omega\left(T^{k} \otimes H^{k}(x \otimes y)\right)-\omega\left(E_{T} \otimes E_{H}(x \otimes y)\right)\right)=0 .
$$

Finally, the density argument shows that $(\mathfrak{H} \otimes \mathfrak{B}, T \otimes H)$ is unique $E_{T} \otimes E_{H^{-}}$ ergodic.

REMARK 3.8. We note that all the results of this section extend the results of $[14,15]$ to uniquely $E$-ergodic and uniquely $E$-weak mixing dynamical systems.

REMARK 3.9. We have to stress that the unique ergodicity of $T \otimes H$ does not imply unique weak mixing of $T$. Indeed, let us consider the following examples. 
Example 3.10. Let $\mathfrak{A}=\mathbb{C}^{2}$ and

$$
T=\left(\begin{array}{ll}
0 & 1 \\
1 & 0
\end{array}\right)
$$

It is clear that $\mathfrak{A}^{T}=\mathbb{C} \mathbb{1}$, so $T$ is ergodic, that is,

$$
\lim _{n \rightarrow \infty} \frac{1}{n} \sum_{k=1}^{n} T^{k}(x, y)=\frac{x+y}{2}(1,1), \quad x, y \in \mathbb{C} .
$$

From the equality

$$
\left|T^{k}(x, y)-\frac{x+y}{2}(1,1)\right|=\left|\frac{x-y}{2}\right|,
$$

we infer that $T$ is not unique weak mixing.

On the other hand, the equality

$$
(\mathfrak{H} \otimes \mathfrak{U})^{T \otimes T}=\{(x, y, y, x): x, y \in \mathbb{C}\}
$$

implies unique $E_{T \otimes T}$-ergodicity of $T \otimes T$.

Example 3.11. Let $\mathfrak{A}=\mathbb{C}^{3}$ and $\mathfrak{B}=\mathbb{C}^{2}$. Consider the a mapping $P: \mathfrak{A} \rightarrow \mathfrak{A}$ given by

$$
P(x, y, z)=(y, x, u y+v z),
$$

where $u, v>0$ and $u+v=1$. It is clear that $P$ is positive and unital. Direct calculations show that $\mathfrak{U}^{P}=\mathbb{C} \mathbb{1}$, which means that $P$ is uniquely ergodic.

Now consider the mapping $P \otimes T$, where $T$ is defined as above. One can see that such a mapping acts as follows:

$$
P \otimes T(\mathbf{x}, \mathbf{y})=(P \mathbf{y}, P \mathbf{x})
$$

where $\mathbf{x}, \mathbf{y} \in \mathfrak{A}$. Hence, we find that

$$
(\mathfrak{A} \otimes \mathfrak{B})^{P \otimes T}=\left\{(\mathbf{x}, P \mathbf{x}): \mathbf{x} \in \mathfrak{A}^{P^{2}}\right\} .
$$

Therefore, from (3.13) one immediately gets

$$
P^{2}(x, y, z)=\left(x, y, u x+u v y+v^{2} z\right) .
$$

Thus, we find that

$$
\mathfrak{A}^{P^{2}}=\left\{\left(x, y, \frac{x+v y}{1+v}\right): x, y \in \mathbb{C}\right\} .
$$

On the other hand, we have $\mathfrak{A}^{P} \otimes \mathfrak{B}^{T}=\mathbb{C} \mathbb{1}$, so that $(\mathfrak{A} \otimes \mathfrak{B})^{P \otimes T} \neq \mathfrak{H}^{P} \otimes \mathfrak{B}^{T}$.

Similarly, reasoning as in Example 3.10 we can show that $P \otimes T$ is uniquely $E_{P \otimes T^{-}}$ ergodic.

Note that, from these examples, we infer the importance of the condition $E_{T \otimes H}=$ $E_{T} \otimes E_{H}$. 


\title{
Acknowledgements
}

The author acknowledges MOHE Grant FRGS11-022-0170. He also thanks the Junior Associate scheme of the Abdus Salam International Centre for Theoretical Physics, Trieste, Italy.

\section{References}

[1] J. Aaronson, M. Lin and B. Weiss, 'Mixing properties of Markov operators and ergodic transformations, and ergodicity of Cartesian products', Israel J. Math. 33(3-4) (1979), 198-224.

[2] B. Abadie and K. Dykema, 'Unique ergodicity of free shifts and some other automorphisms of $C^{*}$-algebras', J. Operator Theory 61 (2009), 279-294.

[3] L. Accardi and F. Mukhamedov, 'A note on noncommutative unique ergodicity and weighted means', Linear Algebra Appl. 430 (2009), 782-790.

[4] D. Avitzour, 'Noncommutative topological dynamical systems, II', Trans. Amer. Math. Soc. 282 (1984), 121-135.

[5] D. Berend and V. Bergelson, 'Mixing sequences in Hilbert spaces', Proc. Amer. Math. Soc. 98 (1986), 239-246.

[6] J. R. Blum and D. L. Hanson, 'On the mean ergodic theorem for subsequences', Bull. Amer. Math. Soc. 66 (1960), 308-311.

[7] R. Duvenhage, 'Joinings of $W^{*}$-dynamical systems', J. Math. Anal. Appl. 343 (2008), 175-181.

[8] F. Fidaleo, 'On strong ergodic properties of quantum dynamical systems', Infin. Dimens. Anal. Quantum Probab. Relat. Top. 12 (2009), 551-556.

[9] F. Fidaleo and F. Mukhamedov, 'Strict weak mixing of some $C^{*}$-dynamical systems based on free shifts', J. Math. Anal. Appl. 336 (2007), 180-187.

[10] F. Fidaleo and F. Mukhamedov, 'Ergodic properties of Bogoliubov automorphisms in free probability', Infin. Dimens. Anal. Quantum Probab. Relat. Top. 13 (2010), 393-411.

[11] L. K. Jones and M. Lin, 'Ergodic theorems of weak mixing type', Proc. Amer. Math. Soc. 57 (1976), 50-52.

[12] H. O. Krengel, Ergodic Theorems (Walter de Gruyter, Berlin, 1985).

[13] A. Łuczak, 'Eigenvalues and eigenspaces of quantum dynamical systems and their tensor products', J. Math. Anal. Appl. 221 (1998), 13-32.

[14] F. Mukhamedov, 'On strictly weakly mixing $C^{*}$-dynamical systems', Funct. Anal. Appl. 27 (2007), 311-313.

[15] F. Mukhamedov, 'On strictly weak mixing $C^{*}$-dynamical systems and a weighted ergodic theorem', Studia Sci. Math. Hungar. 47 (2010), 155-174.

[16] F. Mukhamedov and S. Temir, 'A few remarks on mixing properties of $C^{*}$-dynamical systems', Rocky Mountain J. Math. 37 (2007), 1685-1703.

[17] R. A. Ruan, Introduction to Tensor Products of Banach Spaces (Springer, London, 2002).

[18] M. Takesaki, Theory of Operator Algebras, I (Springer, Berlin, 1979).

[19] S. Watanabe, 'Asymptotic behavior and eigenvalues of dybamical semi-groups on operator algebras', J. Math. Anal. Appl. 86 (1982), 411-424.

[20] L. Zsidó, 'Weak mixing properties of vector sequences', in: The Extended Field of Operator Theory. Containing Lectures of the 15th International Workshop on Operator Theory and its Applications, IWOTA 2004, Newcastle, UK, July 12-16, 2004, Operator Theory: Advances and Applications, 171 (ed. M. A. Dritschel) (Birkhäuser, Basel, 2006), pp. 361-388.

\author{
FARRUKH MUKHAMEDOV, \\ Department of Computational and Theoretical Sciences, \\ Faculty of Science, International Islamic University Malaysia, \\ P.O. Box, 141, 25710, Kuantan, Pahang, Malaysia \\ e-mail: far75m@yandex.ru
}

